Communications in Physics, Vol. 26, No. 2 (2016), pp. 151-157

DOI:10.15625/0868-3166/26/2/8618

\title{
INFLUENCE OF DISULFIDE BRIDGE ON THE STRUCTURAL STABILITY OF HUMAN NEUROGLOBIN: A MOLECULAR DYNAMICS SIMULATION USING LATEST DATA ENTRY
}

\author{
BUI THI LE QUYEN \\ Thai Binh University of Medicine and Pharmacy, \\ 373 Ly Bon, Thai Binh, Vietnam. \\ NGUYEN THI LAM HOAI AND NGO VAN THANH ${ }^{\dagger}$ \\ Institute of Physics, Vietnam Academy of Science and Technology, \\ 10 Daotan, Badinh, Hanoi, Vietnam. \\ ${ }^{\dagger}$ E-mail: nvthanh@iop.vast.ac.vn \\ Received 17 August 2016 \\ Accepted for publication 15 September 2016
}

\begin{abstract}
In this paper, we investigated the role of the disulfide bridge in the structural stability of wild-type human neuroglobin. The classical simulation of the neuroglobin without the disulfide bridge was performed for a long simulation run of 240 ns using a new parameter set of Gromos 96 force field and the latest data entry as the initial topologies. We used the analyzed data of original neuroglobin with the remained disulfide bridge to compare to the ones from this simulation. Our results showed that, the structure of neuroglobin was still very stable although the disulfide bridge was absent. There was only a few residues in $B$ and $C$ helices having a higher mobility. The most interesting result we obtained was that the increasing distance between the distal histidine and heme group could allow oxygen to bind more easily.
\end{abstract}

Keywords: molecular dynamics simulation; neuroglobin; crystal structure of protein.

Classification numbers: 87.14.E-, 87.15.ap, 87.15.H-.

\section{INTRODUCTION}

In the past decade, a new member of globin family, neuroglobin $(\mathrm{Ngb})$ has been discovered in the brain of vertebrates [1]. Ngb is not only expressed in the nervous system of vertebrates [1-4], but in retina, and other nerve tissues as well [5]. Ngb is composed of a single polypeptide of 151 amino acids and a heme prosthetic group which is able to bind to various gaseous ligands $\left(\mathrm{O}_{2}, \mathrm{NO}\right.$ or $\mathrm{CO}$ ) at the central heme iron [6-10].

(C)2016 Vietnam Academy of Science and Technology 
Similar to other members of globin family [11-14], neuroglobin conserves two key histidine residues: a distal histidine His64(E7) and a proximal histidine His96(F8). In the absence of an external ligand, both histidines bind to the heme iron for the ferric $\left(\mathrm{Fe}^{3+}\right)$ and the ferrous $\left(\mathrm{Fe}^{2+}\right)$ forms of $\mathrm{Ngb}$. The histidine His96 is in a hexacoordinated iron because it is near to the coordination sphere of the heme iron atom. Therefore, any external ligand can also compete with the opposite histidine which is His64 (the sixth ligand in helix notation) for the binding of ferrous.

In the structure of neuroglobin, there is an existence of an intramolecular disulfide bridge (S-S bond) in the C-D region involving two cysteine residues Cys46 (C7) and Cys55 (D5). The disulfide bridge which is an interesting feature of Ngb plays an important role in regulating the activity and stability of protein. The experimental studies have shown the increase in $\mathrm{CO}$ rebinding rate and $\mathrm{O}_{2}$ affinity [15-18].

It is well known that the structures of protein should give very important information about its roles and functions in the cell. From those, we can formulate the hypotheses about the functions of particular amino acid of protein. In addition, the experimental results showed that the orientation of the E-helix had been shifted in neuroglobin without the disulfide bridge [19]. Breaking the disulfide bond causes the decrease in affinity and release of $\mathrm{O}_{2}$. This bond could be removed by slowly adding of dithiothreitol (DDT) for about 30 minutes at $37^{\circ} \mathrm{C}$. Depending on the concentration of oxygen, the free cysteines would be oxidized into an intramolecular disulfide bond which consequently increases the $\mathrm{O}_{2}$ affinity and $\mathrm{O}_{2}$ storage simultaneously with increasing the concentration. In this study, we performed the classical simulations in order to investigate the dynamical structures of wild-type human neuroglobin with and without S-S bond. The initial topology of these proteins are generated by the latest data entry. We also used a new parameter set of Gromos 96 force field.

The paper is organized as follows. Section II is devoted to the descriptions of protein preparation and the molecular dynamics (MD) simulations. Results are shown and discussed in Section III. Conclusions are given in Section IV.

\section{MATERIALS AND METHODS}

\section{II.1. Protein data entry}

In the past few years, a new data entry of wild-type human neuroglobin without any residue mutations is solved by using X-ray diffraction at a resolution of $1.74 \AA$ [20]. This data file (PDB entry 4MPM) can be downloaded from the Protein Data Bank. For our simulations, we only used the B-chain because of fewer missing residues at the ends of sequence (MET1, GLY150 and GLU151). The missed residues were manually added by using Swiss-PdbViewer program [21]. For more convenience, in this paper we use the notations Ngb-WSS and Ngb-NSS for wild-type human neuroglobin with and without S-S bond, respectively. Figure 1 visualizes the 3D structure of wild-type human Ngb using original PDB data (4MPM.pdb). Many thanks are due to an excellent molecular visualization program VMD for displaying biomolecular systems using 3D graphics [22].

\section{II.2. Simulation Method}

The molecular dynamics simulations have been performed with the help of Gromacs v5.1.0 computer program [23]. The initial configurations of Ngb were generated by pdb2gmx script 


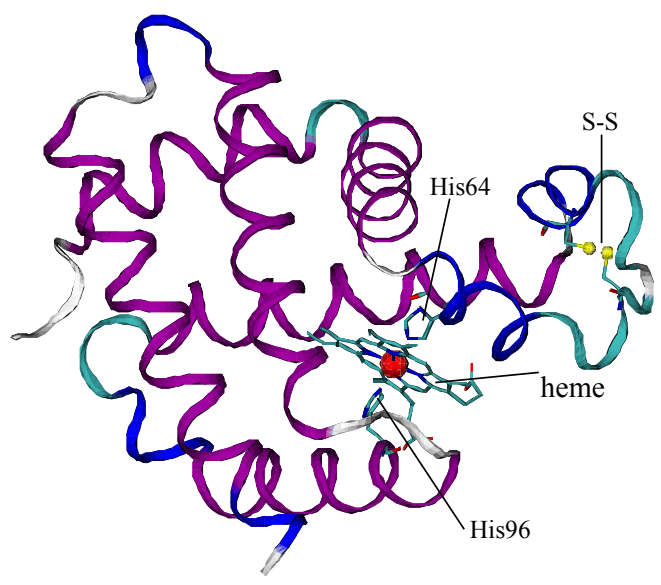

Fig. 1. Ribbon representation of wild-type human Ngb. The Fe atom is highlighted by a red sphere at center of heme group.

including the latest parameter set $54 \mathrm{a} 7$ of Gromos96 force field [24]. Note that, we must choose "-noss" option in this program for removing S-S bond from topological configuration of Ngb-NSS.

The simulations were carried out at constant temperature of $300 \mathrm{~K}$ with the initial velocity of atoms taken randomly from a Maxwellian distribution. The short-range electrostatic and van der Waals cutoffs were $1.4 \mathrm{~nm}$. For the calculation of the long-range interactions, we used the particle mesh Ewald method with a grid spacing of $0.16 \mathrm{~nm}$. We used fourth-order cubic interpolation in order to compute the potentials and forces between grid points. The periodic boundary condition was used.

We placed each protein at center of a cubic box with SPC/E water model [25]. The distance to the edge of the box was chosen at least $1.2 \mathrm{~nm}$. We first performed a short MD simulation for the vacuo minimization. In this process, the maximum number of steps was 50,000 with energy step size equals to 0.01 . Next, the protein solvated with explicit single-point charge water molecules and therefore the total number of atoms was about 40,000. In order to neutralize the net charge of our systems, we added 5 positive ions replacing water molecules with $\mathrm{Na}^{+}$. The solvent was relaxed through a process called energy minimization followed by 500ps of MD runs to ensure that the system had no steric clashes or inappropriate geometry.

The equilibration was performed in an attempt to relax the solvent and ions while keeping the protein atom positions restrained. These steps were conducted under the two ensembles: an $N V T$ (constant Number of particles, Volume, and Temperature) and an NPT (constant Number of atoms, Pressure, and Temperature). The first simulation brought the system to the target temperature of $300 \mathrm{~K}$. The second one was needed to allow the system to found the correct density. Note that, the total MD simulation time was 400ps for each equilibration procedure.

Finally, we run production MD simulations for data collection by $240 \mathrm{~ns}$ at room temperature $(300 \mathrm{~K})$. All the simulations were performed on the CPU (Intel Core i7: 6 cores, 12 threads) for parallel computing with OpenMP. 


\section{SIMULATION RESULTS}

In this section, we show the analyzed results for our system. The MD simulation data of $\mathrm{Ngb}$ with the disulfide bridge (Ngb-WSS) are used as the structural reference for comparison. From this stage, we use the notations "wss" and "nss" for wild-type neuroglobin with and without $\mathrm{S}-\mathrm{S}$ bond. We also use the conventional symbols in the plots for a distinction between two proteins, namely star/circle points. In our calculations, the distance between two atom groups (residue or heme group) is actually the distance between their centers of mass.

Using the trajectory data obtained from MD simulation, we first analyze the root mean square deviation $(R M S D)$ of atomic positions which is the measurement of average distance between the atoms of proteins. The RMSD is a common way to compare the structures of biomolecules, it is defined by

$$
\operatorname{RMSD}(t)=\left[\frac{1}{M} \sum_{i=1}^{N} m_{i}\left\|r_{i}(t)-r_{i}(0)\right\|^{2}\right]^{1 / 2},
$$

where the sum is taken over all the atoms of protein, $M=\sum_{i=1}^{N} m_{i}$ with $m_{i}$ being the mass of atom $i$. $r_{i}(t)$ and $r_{i}(0)$ are the positions of atom $i$ at time frame $t$ and 0 . The initial structure at time $t=0$ is chosen as a reference.

We show in Fig. 2 the changes in RMSD of C-alpha atoms for the structures of $\mathrm{Ngb}$ with and without S-S bond. During the first stage of simulation about $100 \mathrm{~ns}$, the $R M S D$ changes greatly with time. Then, it is reasonably stable around $0.27 \mathrm{~nm}$ and $0.35 \mathrm{~nm}$ for Ngb-NSS (circle points) and Ngb-WSS (star points). Note that, the changes on the order of 1-4 $\AA$ are well acceptable for small proteins. So, this result indicates the structure of Ngb-WSS/NSS is very stable after $\sim 100 \mathrm{~ns}$ (the second stage of simulation).

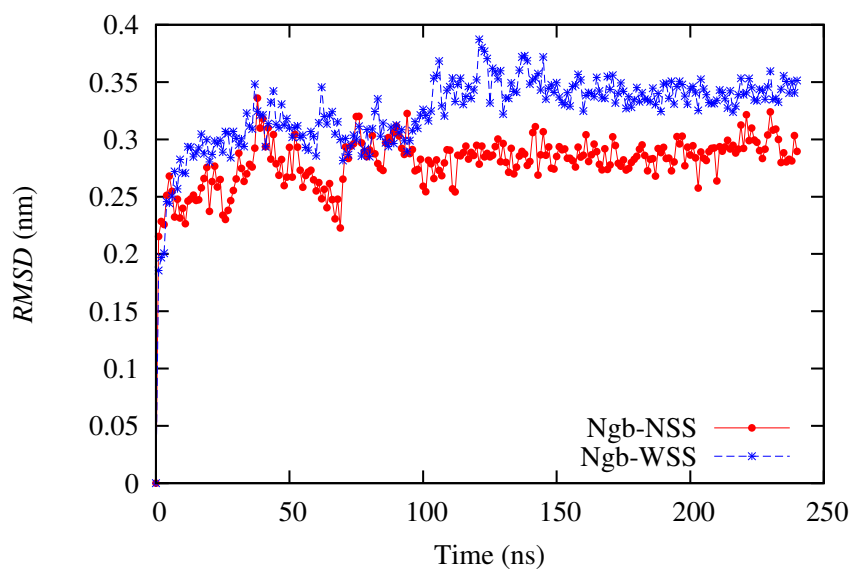

Fig. 2. $R M S D$ of $\mathrm{C}$-alpha atoms after least-square fit to backbone versus simulation times for Ngb-WSS (star points) and Ngb-NSS (circle points).

Now we analyze the time-dependent distance between Cys46 and Cys55 because S-S bond is formed by them in C-D loop region. Let $d_{\mathrm{C} 46-\mathrm{C} 55}^{\mathrm{wss}}$ and $d_{\mathrm{C} 46-\mathrm{C} 55}^{\mathrm{nss}}$ denote the distances between the two cysteine residues of Ngb-WSS and Ngb-NSS. As see in the figure $3 \mathrm{a}$, the distance $d_{\mathrm{C} 46-\mathrm{C} 55}^{\mathrm{wss}}$ 
(star points) is very stable around $0.4 \mathrm{~nm}$ with the existence of S-S bond while the distance $d_{\mathrm{C} 46-\mathrm{C} 55}^{\mathrm{nss}}$ is larger than that of Ngb-WSS (circle points) and greatly fluctuated around $\sim 1.2 \mathrm{~nm}$. The histograms of these distances are demonstrated in Fig. $3 \mathrm{~b}$ for a quantitative comparison.
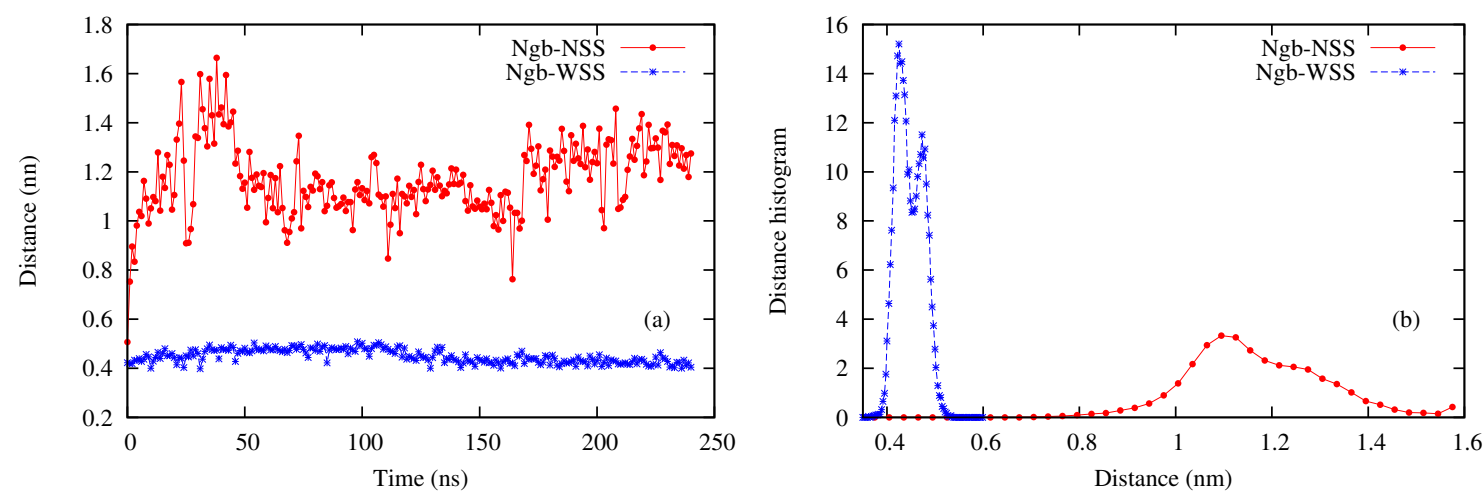

Fig. 3. The time-dependent distances between Cys46 and Cys55 residues (a), and the distance histograms (b), for Ngb-WSS (star points) and Ngb-NSS (circle points).

Next, we investigate the changes in the dynamical properties of heme group when Cys46 and Cys55 residues are unbounded. It is well known that both histidines bind to the heme iron for $\mathrm{Fe}^{3+}$ and $\mathrm{Fe}^{2+}$ forms. Although the distal histidine (His64) appears fixed in the crystal structure, but it can swing out of the heme group to allow oxygen to bind to the iron.
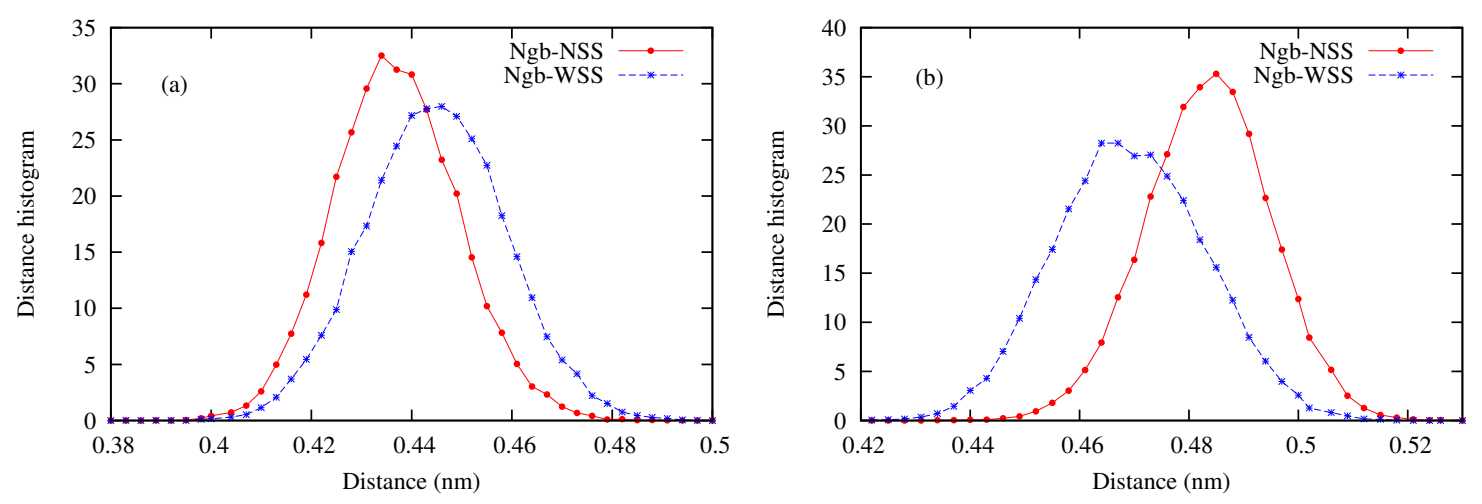

Fig. 4. The distance histograms of pair His96/heme residues (a) and of pair His64/heme residues (b), for Ngb-WSS (star points) and Ngb-NSS (circle points).

As observed in Fig. 4, the peaks of the distance histograms are located around $0.446 \mathrm{~nm}$ (star points) and $0.434 \mathrm{~nm}$ (circle points) for the pair His96/heme; at $0.466 \mathrm{~nm}$ (star points) and at $0.485 \mathrm{~nm}$ (circle points) for the pair His64/heme. Those indicate that the heme group of Ngb-NSS drifted toward the proximal histidine His96. Besides, there is a rather difference of the distances between two histidine residues, the one of Ngb-NSS is larger. Therefore, the distance from the heme group to distal histidine of Ngb-NSS is larger than that of Ngb-WSS. 


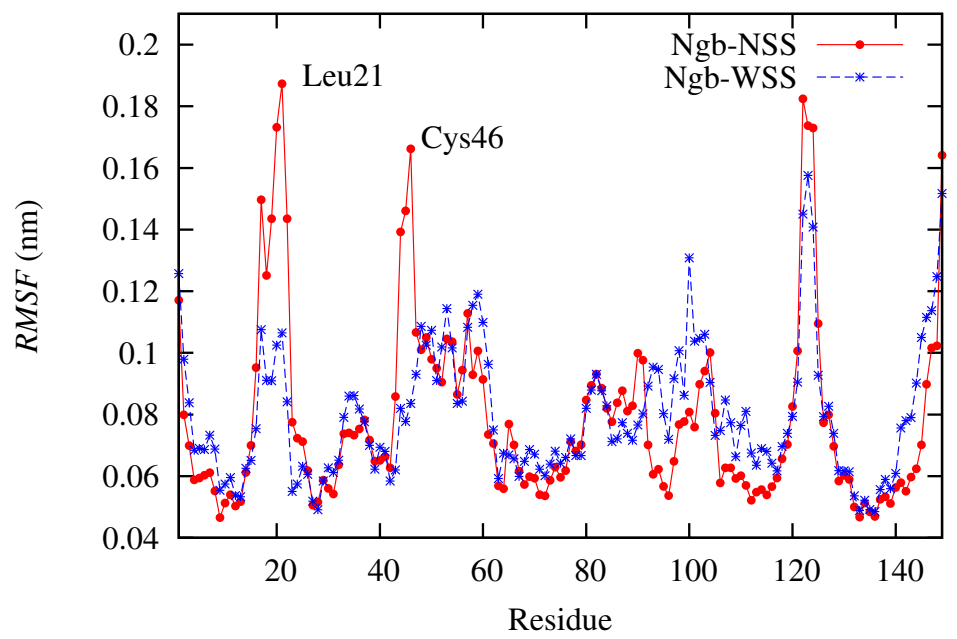

Fig. 5. Root mean square fluctuation of backbones for Ngb-WSS (star points) and NgbNSS (circle points).

We consider another quantity called the atomic root mean square fluctuation ( $R M S F$, i.e. standard deviation) which is the simplest and most used property for comparing the dynamics proteins. It is also a measure of the average atomic mobility. The RMSF of atomic positions in the trajectory can be written in the form

$$
\operatorname{RMSF}(i)=\sqrt{\frac{1}{T} \sum_{t=0}^{T}\left[\boldsymbol{r}_{i}(t)-\overline{\boldsymbol{r}}_{i}\right]},
$$

in which $\overline{\boldsymbol{r}}_{i}$ is the average position of atom $i$, the sum is taken over all the simulation time steps.

The RMSF of backbones for Ngb-WSS and Ngb-NSS are shown in Fig. 5. In the case of $\mathrm{Ngb}$ without S-S bond, we find two peaks of RMSF around the residues Leu21 and Cys46 with highest amplitude fluctuations. As seen in this plot, the B and $\mathrm{C}$ helices fluctuated the most during the simulation.

\section{CONCLUSIONS}

We have shown in this paper the role of the S-S bridge formation in protein folding of wild-type human Ngb. We used the SCP/E water model and the most recent Gromos96 54a7 force field. Furthermore, the newest PDB entry 4MPM has been used to create input coordinates and topology for gromacs program. The results are obtained with the highly performing MD simulation for a long period of $240 \mathrm{~ns}$ at $300 \mathrm{~K}$. We compared the structural changes in wild-type human neuroglobin with and without the disulfide bridge.

In the case of Ngb without the S-S bridge, Cys46 and Cys55 residues in the C and D helices drift apart from each other. Additionally, the residues in these helices have a greatly high mobility. On the other hand, the removal of S-S bond results in reducing distance between His96 residue and heme group, and in increasing distance between pair of His64/heme simultaneously. Therefore, 
the histidine His64 is far away from the heme group, it may easily leave and empty space for binding functional ligand.

We showed that the structures of Ngb are very stable after $100 \mathrm{~ns}$ simulation no matter there is a presence of S-S bond or not. So, we should perform the simulations long enough for high accurate analyzes. The simulation results which were presented here can also serve as a validation of new PDB entry (4MPM).

\section{ACKNOWLEDGMENT}

This work was supported by the Lotus program, Grand No. 45/2012/HD-NDT.

\section{REFERENCES}

[1] T. Burmester, B. Weich, S. Reinhardt, and T. Hankeln, Nature 407 (2000) 520.

[2] M. Brunori and B. Vallone, FASEB J. 20 (2006) 2192.

[3] M. Brunori and B. Vallone, Cell. Mol. Life Sci. 64 (2007) 1259.

[4] K. Nienhaus and G. U. Nienhaus, IUBMB Life 59 (2007) 490.

[5] T. Burmester and T. Hankeln, J. Exp. Biol. 212 (2009) 1423.

[6] S. Dewilde, L. Kiger, T. Burmester, T. Hankeln, V. Baudin-Creuza, T. Aerts, M. C. Marden, R. Caubergs and L. Moens, J. Biol. Chem. 276 (2001) 38949.

[7] A. Pesce, S. Dewilde, M. Nardini, L. Moens, P. Ascenzi, T. Hankeln, T. Burmester, and M. Bolognesi, Structure 11 (2003) 1087-1095.

[8] A. Pesce, S. Dewilde, M. Nardini, S. Dewilde, L. Moens, T. Hankeln, T. Burmester, P. Ascenzi and M. Bolognesi, IUBMB Life 56 (2004) 657.

[9] B.Vallone, K. Nienhaus, M. Brunori, and G. U. Nienhaus, Proteins 56 (2004) 85-92.

[10] A. Bocahut, S. Bernad, P. Sebban and S. Sacquin-Mora, J. Phys Chem B 113 (2009) 16257.

[11] F. Hoppe-Seyler, Über die oxydation in lebendem blute Med-chem Untersuch Lab 1 (1866) 133.

[12] M. Brunori, Rend. Fis. Acc. Lincei 21 (2010) 335.

[13] N. Kawada, D. B. Kristensen, K. Asahina, K. Nakatani, Y. Minamiyama, S. Seki and K. Yoshizato, The Journal of Biological Chemistry 276 (2001) 25318.

[14] T. Burmester, B. Ebner, B. Weich and T. Hankeln, Molecular Biology and Evolution 19 (2002) 416.

[15] D. Hamdane et al., J. Biol. Chem. 278 (2003) 51713.

[16] D. Hamdane, L. Kiger, S. Dewilde, B.N. Green, A. Pesce, J. Uzan, T. Burmester, T. Hankeln, M. Bolognesi, L. Moens and M.C. Marden, Micron 35 (2004) 59.

[17] E. Vinck, S. Van Doorslaer, S. Dewilde and L. Moens, J. Am. Chem. Soc. 126 (2004) 4516.

[18] L. Astudillo, S. Bernad, V. Derrien, P. Sebban and J. Miksovska, Biophys. J. 99 (2010) L16-L18.

[19] D. Hamdane et. al., J. Biol. Chem. 278 (2003) 51713.

[20] B. G. Guimarães, D. Hamdane, C. Lechauve, M. C. Marden and B. Golinelli-Pimpaneau, Acta Cryst. D 70 (2014) 1005.

[21] N. Guex and M.C. Peitsch, Electrophoresis 18 (1997) 2714.

[22] W. Humphrey, A. Dalke and K. Schulten, J. Molec. Graphics, 14 (1996) 33-38.

[23] S. Pronk, S. Páll, R. Schulz, P. Larsson, P. Bjelkmar, R. Apostolov, M.R. Shirts, J.C. Smith, P.M. Kasson, D. van der Spoel, B. Hess, E. Lindahl, Bioinformatics 29(7), 845854 (2013); M.J. Abraham, D. van der Spoel, E. Lindahl, B. Hess, and the GROMACS development team, "GROMACS User Manual version 5.1", www.gromacs.org (2015).

[24] N. Schmid, A.P. Eichenberger, A. Choutko, S. Riniker, M. Winger, A.E. Mark and W.F. van Gunsteren, Eur. Biophys. J. 40 (2011) 843-856.

[25] H. J. C. Berendsen, J. R. Grigera and T. P. Straatsma, J. Phys. Chem. 91 (1987) 62696271. 
